\title{
Diffusion Reflection Method for Early Detection of Oral Squamous Cell Carcinoma Specifically Targeted by Circulating Gold-Nanorods Bio-Conjugated to Anti-Epidermal Growth Factor Receptor
}

This article was published in the following Dove Press journal:

International Journal of Nanomedicine

\author{
Shiran Sudri' \\ Hamootal Duadi ${ }^{2}$ \\ Florin Altman' \\ Irit Allon ${ }^{3}$ \\ Ariel Ashkenazy (iD $)^{2}$ \\ Ruchira Chakraborty ${ }^{2}$ \\ Ilya Novikov ${ }^{4}$ \\ Dror Fixler ${ }^{2, *}$ \\ Abraham Hirshberg (D) ${ }^{1, *}$ \\ 'Department of Oral Pathology and Oral \\ Medicine, The Maurice and Gabriela \\ Goldschleger School of Dental Medicine, \\ Tel Aviv University, Tel Aviv, Israel; \\ ${ }^{2}$ Faculty of Engineering and the Institute \\ of Nanotechnology and Advanced \\ Materials, Bar llan University, Ramat Gan, \\ 5290002, Israel; ${ }^{3}$ Institute of Pathology, \\ Barzilai Medical Center, Ben Gurion \\ University of the Negev, Beer Sheba, \\ Israel; ${ }^{4}$ Gertner Institute for \\ Epidemiology and Health Policy Research, \\ Ramat Gan, Israel
}

*These authors contributed equally to this work

Correspondence: Abraham Hirshberg Department of Oral Pathology and Oral Medicine, The Maurice and Gabriela Goldschleger School of Dental Medicine,

Tel Aviv University, 4 Klachkin Street, Ramat-Aviv, Tel-Aviv, 613900I, Israel

Tel +972 3-6409303

Fax +972 3-6409250

Email hirshmd@tauex.tau.ac.il
Background: Translation of nanomedical developments into clinical application is receiving an increasing interest. However, its use for oral squamous cell carcinoma (OSCC) diagnosis remains limited. We present an advanced nanophotonic method for oral cancer detection, based on diffusion reflection (DR) measurements of gold-nanorods bio-conjugated to anti-epidermal growth factor receptor (C-GNRs) specifically attached to OSCC cells.

Objective: To investigate in a rat model of oral carcinogenesis the targeting potential of C-GNRs to OSCC by using the DR optical method.

Materials and Methods: OSCC was induced by the carcinogen 4-nitroquinoline-N-oxide (4NQO). C-GNRs were introduced locally and systemically and DR measurements were recorded from the surface of the rat tongue following illumination with red laser beam. Rats were divided into experimental and control groups. The results were compared with the histologic diagnosis.

Results: A total of 75 Wistar-derived rats were enrolled in the study. Local application did not reveal any statistical results. DR measurements following intravenous injection of C-GNRs revealed a significant increase in light absorption in rats with OSCC compare with rats without cancer ( $\mathrm{p}<0.02$, sensitivity $100 \%$, specificity $89 \%$ ). In addition, absorption of light increased significantly in cases of severe dysplasia and cancer (high risk) compared to rats without cancer and rats with mild dysplasia (low risk) ( $86 \%$ sensitivity and $89 \%$ specificity, AUC $=0.79$ ).

Conclusion: Combining nanotechnology and nanophotonics for in vivo diagnosis of OSCC serves as additional tier in the translation of advanced nanomedical developments into clinical applications. The presented method shows a promising potential of nanophotonics for oral cancer identification, and provides support for the use of C-GNRs as a selective drug delivery.

Keywords: oral cancer, squamous cell carcinoma, gold nanoparticles, anti-EGFR, nanophotonic, cancer detection

\section{Plain Language Summary}

Translation of nanomedical developments into clinical application is receiving increasing interest in recent years, with actively targeted nanoparticles being studied as a diagnostic tool and as a selective drug carriage.

We present an advanced nanophotonic method for cancer detection. The method is based on measuring the reflected light from a tissue (diffusion reflection-DR) following the introduction of gold nanoparticles (GNPs). As GNPs absorb light in the red to infra-red spectrum, illumination with red color laser beam will yield a decrease in the reflected light compared to a tissue without gold nanoparticles. 
Head and neck cancers including oral cancer exhibit an increased expression of epidermal growth factor receptor (EGFR) on their cell membrane. Specific attachment of GNPs to oral cancer cells is achieved by binding the GNPs with antibodies against EGFR and once injected systemically they are attracting the oral cancer site and are easily detected by using the DR photonic method.

In the present study, we investigated in a rat model of oral carcinogenesis the targeting potential of systemically administration of GNPs to oral cancer and the detection of the cancerous lesion using the DR optical method. The results are promising, DR measurements recorded from the surface of the tongue following intravenous injection of GNPs revealed a significant increase in light absorption in rats with oral cancer compare with rats without cancer.

Combining nanotechnology and nanophotonics for in vivo diagnosis of oral cancer serves as an additional tier in the translation of advanced nanomedical developments into clinical applications. The presented method shows a promising potential of nanophotonics for oral cancer identification and provides support for the use of C-GNRs as a selective drug delivery.

\section{Introduction}

Translation of nanomedical developments into clinical application is receiving increasing interest in recent years, with actively targeted nanoparticles being studied as a diagnostic tool and as a selective drug carriage specifically to cancerous sites. ${ }^{1-4}$ Despite the plethora of studies published in the literature, the clinical use of nanoparticlebased agents used for in vivo oral cancer diagnosis and treatment remains limited. ${ }^{5-7}$

Oral squamous cell carcinomas (OSCC) arising from the mucosal surface of the oral cavity are among the ten leading cancers worldwide with about 50\% 5-years survival rate mainly because of late detection. ${ }^{8,9}$ Several adjunctive tests have been applied for early diagnosis, however, none has been recommended for routine clinical use to replace clinical oral examination. ${ }^{10,11}$

Nanoparticles have been studied and applied in the field of tumor diagnosis and treatment and have attracted attention as contrast agents to enhance imaging of oral cancer, $6,7,12-15$ yet, these methods do not affect clinical practice. Due to the remarkable properties of gold nanoparticles, they are considered as a potential tool for cancer diagnosis and drug delivery applications.

Nanophotonics has emerged as an innovative way in the field of medicine mainly to detect and imaging cancer utilizing the unique light absorption properties of goldnanorods (GNRs) in the red to infra-red spectrum. ${ }^{16,17}$
GNRs bio-conjugated to anti-epidermal growth factor receptor (C-GNR) monoclonal antibodies are supposed to attach exclusively to OSCC cells expressing high concentration of EGFR, making them an ideal target for C-GNRs. ${ }^{18-21}$ We have recently described on paraffinembedded tissue sections of OSCC incubated with C-GNRs, a significant increase in light absorption using diffusion reflection (DR) measurements following illumination with a red color laser beam. ${ }^{16,17}$ In vivo studies are needed to investigate the accuracy and applicability of these nanomedical methods in clinical use.

The main perspectives of the study were to investigate in a rat model of oral carcinogenesis the potential of the DR optical method to detect OSCC specifically targeted by $\mathrm{C}-\mathrm{GNR}$ and to discriminate cancerous and high-risk lesions from non-cancerous low-risk lesions.

\section{Materials and Methods}

\section{Carcinogen-Induced Squamous Cell Carcinoma in Rat Tongue}

The protocol involving animals was approved by the Institutional Animal Care and Use Committee of the Tel Aviv University, and the Ministry of Health, authorization No. 01-16-107, (The procedures described are according to National Research Council (US), Guide for the Care and Use of Laboratory Animals. 8th edition. Washington (DC): National Academies Press (US); 2011). The study followed strict criteria for the use of minimum number of rats.

A rat model of oral carcinogenesis using 4-nitroquinoline-N-oxide (4NQO) administered via drinking water is a well-known model simulating the development of oral cancer in humans. ${ }^{23-25}$ The study group consisted of Wistar-derived male rats weighing about $200 \mathrm{~g}$. Rats were fed standard pellets and water ad libitum. 4NQO (SigmaAldrich) dissolved in dimethyl sulfoxide (Sigma-Aldrich) and then in tap water to a final concentration of $0.001 \%$. Water was replaced once a week with a freshly prepared solution. Bottles of drinking water were shielded with aluminum foil. Animals were carefully inspected daily.

\section{The Experimental Set-Up}

GNRs sized $15 \times 50 \mathrm{~nm}$, were selected as targeted contrast agents because of the high scattering and absorption properties compared to gold nanospheres. ${ }^{26,27}$ Rod shape nanoparticles in the circulation have an increased tendency to migrate toward vessel walls and extravasate easily into the 
tumor microenvironment. ${ }^{5,28,29}$ Conjugated Gold nanorods with anti-EGFR monoclonal antibody Cetuximab (C-GNR) were used to target OSCC (NanoPartz ${ }^{\mathrm{TM}}$ Canada). The extinction coefficient spectrum of the GNRs was determined using a spectrophotometer, and the resultant extinction peak was $650 \mathrm{~nm}$.

C-GNRs were introduced in three routes, external application to the surface of the tongue, direct submucosal injection and systemic injection via the tail vein. A C-GNR concentration of $6 \mathrm{mg} / \mathrm{mL}$ was chosen to be used for the study; $50 \mu \mathrm{L}$ in the external application and in the submucosal injection, and $200 \mu \mathrm{L}$ for the intravenous administration. Following an interval of 96 hours, rats were sacrificed and sent for DR measurements. The concentration of the injected C-GNRs and the interval for the DR measurements were investigated preliminarily for the best DR results.

Table 1 depicts the various study groups. Rats were divided into normal healthy rats and rats treated with 4NQO and whether or not C-GNRs were injected. Rats treated with 4NQO were divided histologically according to severity of the dysplastic changes (mild dysplasia, moderate and severe dysplasia and invasive cancer).

The experimental setting of the systemically injected C-GNRs is presented in Figure 1. In all rats, 3-5 DR measurements were recorded for each area, in the posterior dorsum of the tongue, an area where cancer might develop, and from the anterior tongue.

After DR recording, a mid-lingual incision was made and specimens were fixed in $10 \%$ buffered formalin and embedded in paraffin. Two consecutive $5 \mu \mathrm{m}$ sections were cut on a glass slide. One slide was stained with Hematoxylin and Eosin (H\&E) to evaluate the histologic diagnosis, and one unstained slide was submitted for hyper-spectral imaging.

\section{Diffusion Reflection (DR) Measurements}

The noninvasive optical technique was used for the DR measurements, as has been previously reported. ${ }^{30-32}$ Briefly, the set-up includes an excitation source, which illuminates the tissue with a laser diode with wavelengths of $650 \mathrm{~nm}$, and a photodetector recording the reflected intensity $(\Gamma)$ in different distances $(\rho)$ from the light source. The spatial intensities present a clear difference between the reflectance of the regular tissue, while the intensities from the normal tissue are high and decrease rapidly toward zero intensity for tissues with GNRs. The low intensity, as well as its rapid decrease, is due to the absorption of light by the GNRs, indicating the presence of GNRs in the cancer. In our previous study, ${ }^{33}$ we have introduced the mathematical correlation, which best fit between the optical properties of the irradiated tissue and its DR profile measured by our optical set up:

$$
\ln (\rho \Gamma(\rho))=C_{1}-\mu \rho
$$

while $\mu$ is the effective attenuation coefficient, given by:

$$
\mu=\sqrt{3 \cdot \mu_{a} \mu_{s}^{\prime}}
$$

when $\rho$ is the distance between the light source and the detection, $\Gamma$ is the reflectance intensity, $\mathrm{C}_{1}$ is a constant depends on the optical set up apertures, and $\mu$ is the effective attenuation coefficient, which depends on $\mu_{\mathrm{a}}$ and $\mu_{\mathrm{s}}$, the absorption and the reduced scattering coefficients of the irradiated sample, respectively. In order to extract $\mu$, we are presenting the results in the logarithmic form $\ln (\rho \Gamma(\rho))$.

The reflected intensity $\Gamma(\rho)$, presenting as units of Volt per $\mathrm{mm}$, was collected using a digital scope (Agilent Technologies, Mso7034a, Santa Clara, CA, USA) and data processed using the LabView program. ${ }^{34}$

The resultant intensity $(\Gamma)$ is plotted as the logarithm of the product between the distance and the reflectance versus the

Table I The Study Groups. Rats Were Divided According to the Histologic Diagnosis and Whether or Not C-GNRs Were Injected. Rats Treated with 4NQO Were Divided According to Various Dysplastic Changes (Hyperplasia, Mild Dysplasia, Moderate to Severe Dysplasia and Cancer)

\begin{tabular}{|l|c|c|c|c|c|c|}
\hline Group & A & B & C & D & E & F \\
\hline Rats & Healthy rats & 4NQO treated & Healthy rats & 4NQO treated & 4NQO treated & 4NQO treated \\
\hline Histology & Normal mucosa & OSCC & Normal mucosa & $\begin{array}{c}\text { Hyperkeratosis } \\
\text { Mild dysplasia }\end{array}$ & $\begin{array}{c}\text { Moderate To Severe } \\
\text { dysplasia }\end{array}$ & OSCC \\
\hline $\begin{array}{l}\text { Injected } \\
\text { C-GNRs }\end{array}$ & - & - & + & + & + \\
\hline
\end{tabular}




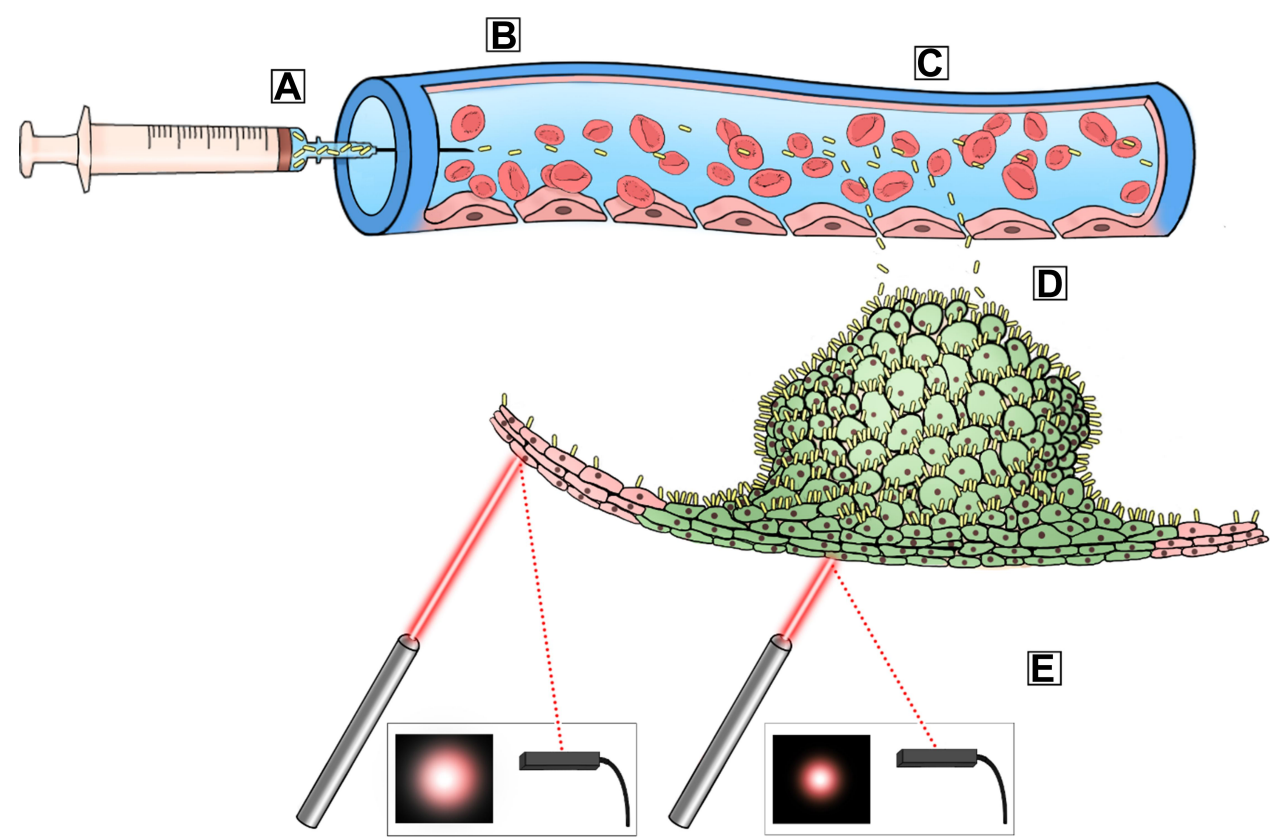

Figure I Experimental setup. (A) Injection of GNRs to the rat's tail vein. (B) GNRs are flowing in the rat's circulation. (C, D) At the area of the tumor there Is higher penetration of the blood vessels, since the tumor over express EGFR on the cell membrane, GNRs are attached to the tumor in high concentration. (E) DR measurements are being recorded; the absorption of the red color laser beam is higher at the area of the tumor where there is high concentration of GNRs. It is also possible to see the illustration of the cell membrane with the attached GNRs (all images used in Figure I are original).

distance $(\rho)$. Therefore, for tissue with high absorption of light, the graph slope is steeper. For convenience, the slope obtained from reflectance measurements is given as absolute value.
An example of how DR results are analyzed is presented in Figure 2, the reflected light is measured and the logarithm representation of the measurement according to Eq. 1 is

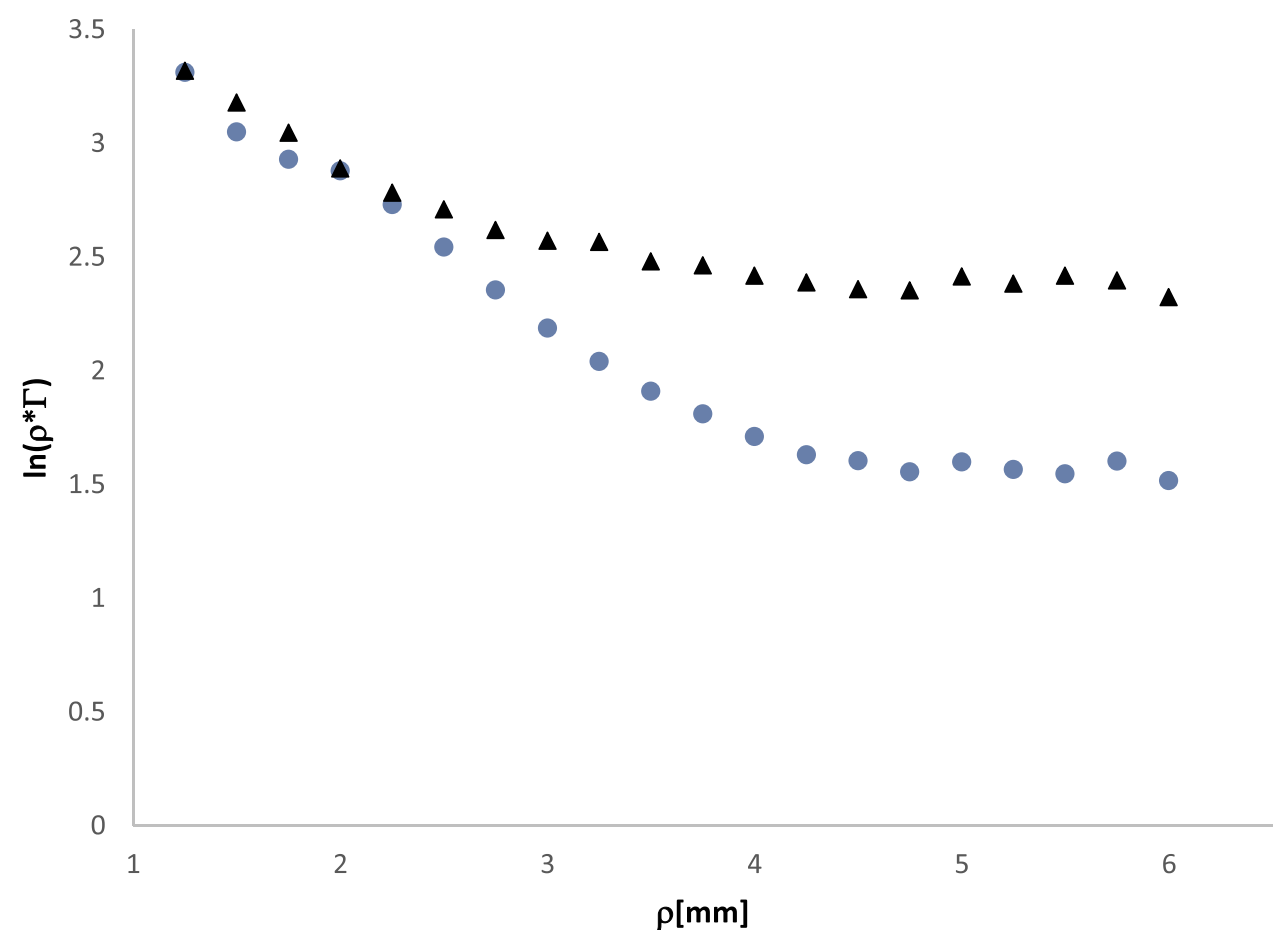

Figure 2 Example of logarithm representation of DR measurements. The logarithmic product between the distance and the reflectance $(\Gamma)$ versus the distance $(\rho)$. DR profile of a healthy rat (group A - black triangles) has a lower absolute slope compared to a rat with cancer (group F - blue circles). 
presented. The slope of a rat with cancer (group F - blue circles) is higher than the slope of a healthy rat (group C black triangles) due to the higher concentration of C-GNRs.

\section{Hyperspectral Microscopy}

A hyperspectral imaging system (Nuance, Cri, Woburn, MA, USA) was used to demonstrate the presence of the GNRs in the tissue to validate the results of the DR measurements.

Formalin-fixed paraffin-embedded tissue sections were used. After deparaffinization, the area of interest, the area corresponding to cancer, was marked on the slide and viewed under hyperspectral microscopy. The reflectance intensities from each sample were captured and analyzed at $650 \mathrm{~nm}$, according to the GNRs excitation peak.

\section{Statistical Analysis}

Of the various DR measurements, we found that the lowest DR values, which correspond to the maximum absorption of light (highest DR slope values) gave the biggest Area Under ROC Curve (AUC), i.e. the best discrimination. Therefore, we present the results for the absolute values of minimum DR measurements.

We used exact Mann-Whitney (MW) and KruskalWallis (KW) tests to compare DR in two or more groups.

The first analysis was to find whether the injected C-GNR accumulated in the cancerous site by analyzing the DR values between rats with cancer (group F) against control rats (group C). Then, we analyzed the discrimination ability of the DR measurements after C-GNRs injection between rats with lowrisk lesions (control and mild dysplasia combined, groups $\mathrm{C}$ and $\mathrm{D}$ ) with rats with high-risk lesions (moderate to severe dysplasia and OSCC combined, groups E and F). We used Cuzick non-parametric test for trend, to test if the absorption of light increases with the stage of tumor evolution. We applied ROC analysis to estimate the test in whole. The general discrimination ability was expressed using Area Under Curve
(AUC). We fixed the cut-point that reached the maximum sum of Sensitivity and Specificity in comparison between groups $\mathrm{C}$ and $\mathrm{D}$ versus groups $\mathrm{E}$ and $\mathrm{F}$. After fixing the cut-point we compared the results by the Exact Fisher (EF) test for $2 \times 2$ tables. We also calculated the Diagnostic Odds Ratio (DOR) that is defined as

$($ sensitivity $) *($ specificity $) /((1-$ sensitivity $) *(1-$ specificity $))$.

OSCC developed in all rats in the posterior dorsum of the tongue, therefore, we compared the DR in the posterior and anterior parts using exact paired Mann-Whitney (Wilcoxon) test.

The whole analysis was repeated after exclusion potential outliers from measurement in a region if there were 3 measurements in the region. The results were quite similar and are not shown.

The analysis was done using STATA 16 SE software. All tests were two-sided. P-values under 0.05 were described as significant.

\section{Results}

A total of 75 rats were enrolled in the study, of these 8 rats for the surface application, 13 rats for the direct submucosal injection and 29 rats for the intravenous application. In addition, 15 rats were used to determine the concentration and the interval for the DR measurements. Ten rats expired during the study. 4NQO was administered for 16 weeks, and rats were sacrificed at two-week interval starting at week 10 until week 26 . The severity of the dysplastic changes increased with time. Cancer was initially found at week 14 and the experiment terminated at week 26. Cancer developed in the posterior dorsum of the tongue in all rats (Figure 3A and $\mathrm{B}$ ), various dysplastic changes were found throughout the mucosa of the tongue.
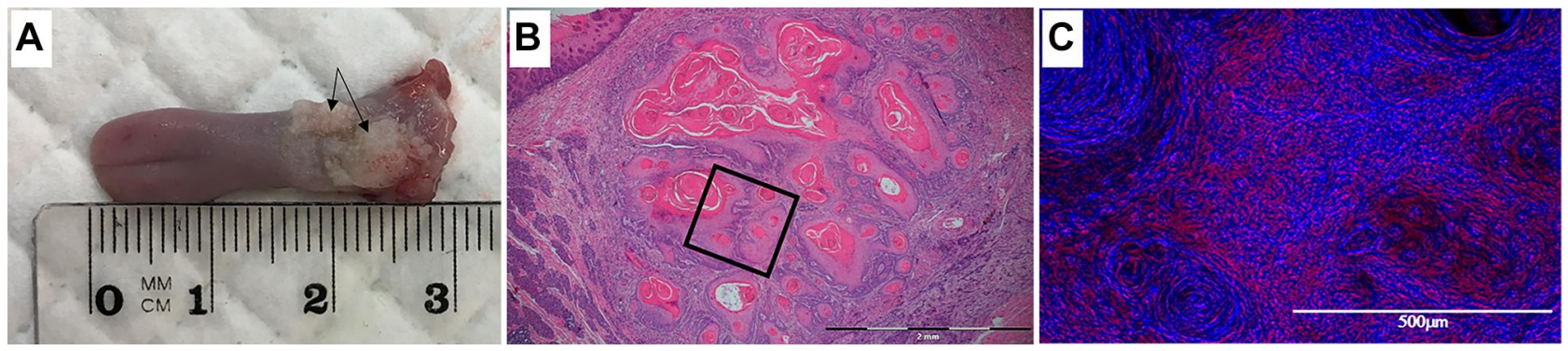

Figure 3 (A) Cancer in the posterior dorsum of the tongue (arrow), (B) Low-power photomicrograph showing typical well differentiated squamous cell carcinoma exhibiting deep invasion (hematoxylin and eosin stain, original magnification $\times 40$ ), (C) hyperspectral imaging, GNRs are shown as red dots, higher magnification of the marked area shown in B (original magnification $\times 200$, scale bar is $500 \mu \mathrm{m}$ ). 


\section{DR Measurements}

External application yielded no results; the C-GNRs could not penetrate the keratin barrier. In the direct submucosal injection, DR measurements could not separate cancerous from noncancerous lesions as the C-GNRs accumulated at the site of injection and did not spread in the cancer. Therefore, only the results of the systemic administration are presented (Table 2). The experimental group consisted of 9 rats with cancer (group F); and 9 rats with various dysplastic lesions (group D and E). Control rats included 6 rats without the administration of C-GNRs (group A and B), and 5 rats without cancer, but with systemically administration of C-GNRs (group C).
C-GNRs were injected systemically to all rats in groups $\mathrm{C}$, $\mathrm{D}, \mathrm{E}$, and $\mathrm{F}$, and DR measurements were recorded in all rats.

Summary of the data is presented in Table 2 and Figures 2 and 4. The highest absolute DR slope values were recorded in rats with cancer (group F) and in rats with moderate to severe dysplasia (group E). The lowest values were recorded in rats with hyperkeratosis and mild dysplasia, and in all of the control rats reflecting the low concentration of EGFR (see Statistics).

Figure 3C presents the hyperspectral image, C-GNRs are clearly demonstrated as red dots concentrated at the cancerous area.

Table 2 Summary of the Data. Experimental and Control Rats, Diagnosis and Absolute Minimum DR Measurements for Anterior and Posterior Dorsum of the Tongue

\begin{tabular}{|c|c|c|c|c|}
\hline Rat & Group & Diagnosis & DR Measurements Posterior Dorsum & DR Measurements Anterior Dorsum \\
\hline I. & \multirow[t]{3}{*}{ A } & Control with no gold & 0.1773 & 0.24 \\
\hline 2. & & Control with no gold & 0.2216 & 0.2464 \\
\hline 3. & & Control with no gold & $0.24 I$ & 0.234 \\
\hline 4. & \multirow[t]{3}{*}{ B } & Control - Cancer with no C-GNR & 0.267 & 0.219 \\
\hline 5. & & Control - Cancer with no C-GNR & 0.218 & 0.164 \\
\hline 6. & & Control - Cancer with no C-GNR & 0.2195 & 0.219 \\
\hline 7. & \multirow[t]{5}{*}{ C } & Control healthy + C-GNR & 0.182 & 0.26 \\
\hline 8. & & Control healthy + C-GNR & 0.1834 & 0.24 \\
\hline 9. & & Control healthy + C-GNR & 0.2187 & 0.143 \\
\hline 10. & & Control healthy + C-GNR & 0.2262 & 0.2897 \\
\hline II. & & Control healthy + C-GNR & 0.203 & 0.167 \\
\hline 12. & \multirow[t]{4}{*}{$\mathrm{D}$} & Hyperplasia-Mild dysplasia & 0.1462 & 0.0987 \\
\hline 13. & & Hyperplasia-Mild dysplasia & 0.2413 & 0.2092 \\
\hline 14. & & Hyperplasia-Mild dysplasia & 0.3645 & 0.2782 \\
\hline I5. & & Hyperplasia-Mild dysplasia & 0.2131 & 0.1936 \\
\hline 16. & \multirow[t]{5}{*}{$\mathrm{E}$} & Moderate- severe dysplasia & 0.2875 & 0.2998 \\
\hline 17. & & Moderate-severe dysplasia & 0.3193 & 0.3314 \\
\hline 18. & & Moderate-severe dysplasia & 0.277 I & 0.2584 \\
\hline 19. & & Moderate-severe dysplasia & 0.1668 & 0.2311 \\
\hline 20. & & Moderate-severe dysplasia & 0.3816 & 0.2349 \\
\hline 21. & \multirow[t]{9}{*}{$\mathrm{F}$} & Carcinoma & 0.3295 & 0.234 \\
\hline 22. & & Carcinoma & 0.3459 & 0.1278 \\
\hline 23. & & Carcinoma & 0.144 & 0.086 \\
\hline 24. & & Carcinoma & 0.2946 & 0.0747 \\
\hline 25. & & Carcinoma & 0.2873 & 0.2374 \\
\hline 26. & & Carcinoma & 0.3868 & 0.1906 \\
\hline 27. & & Carcinoma & 0.3139 & 0.3 \\
\hline 28. & & Carcinoma & 0.2489 & 0.1273 \\
\hline 29. & & Carcinoma & 0.2999 & 0.29 \\
\hline
\end{tabular}

Note: C-GNRs were injected in all experimental rats (group D, E, F) and in control rats group C.

Abbreviations: A, group A control healthy rats with no C-GNR injected; B, group B control rats with cancer with no C-GNR injected; C, group C control healthy rats with gold injected; D, group D experimental rats - hyperplasia/mild dysplasia; E, group E experimental rats - moderate/severe dysplasia; $F$, group F experimental rats - OSCC. 


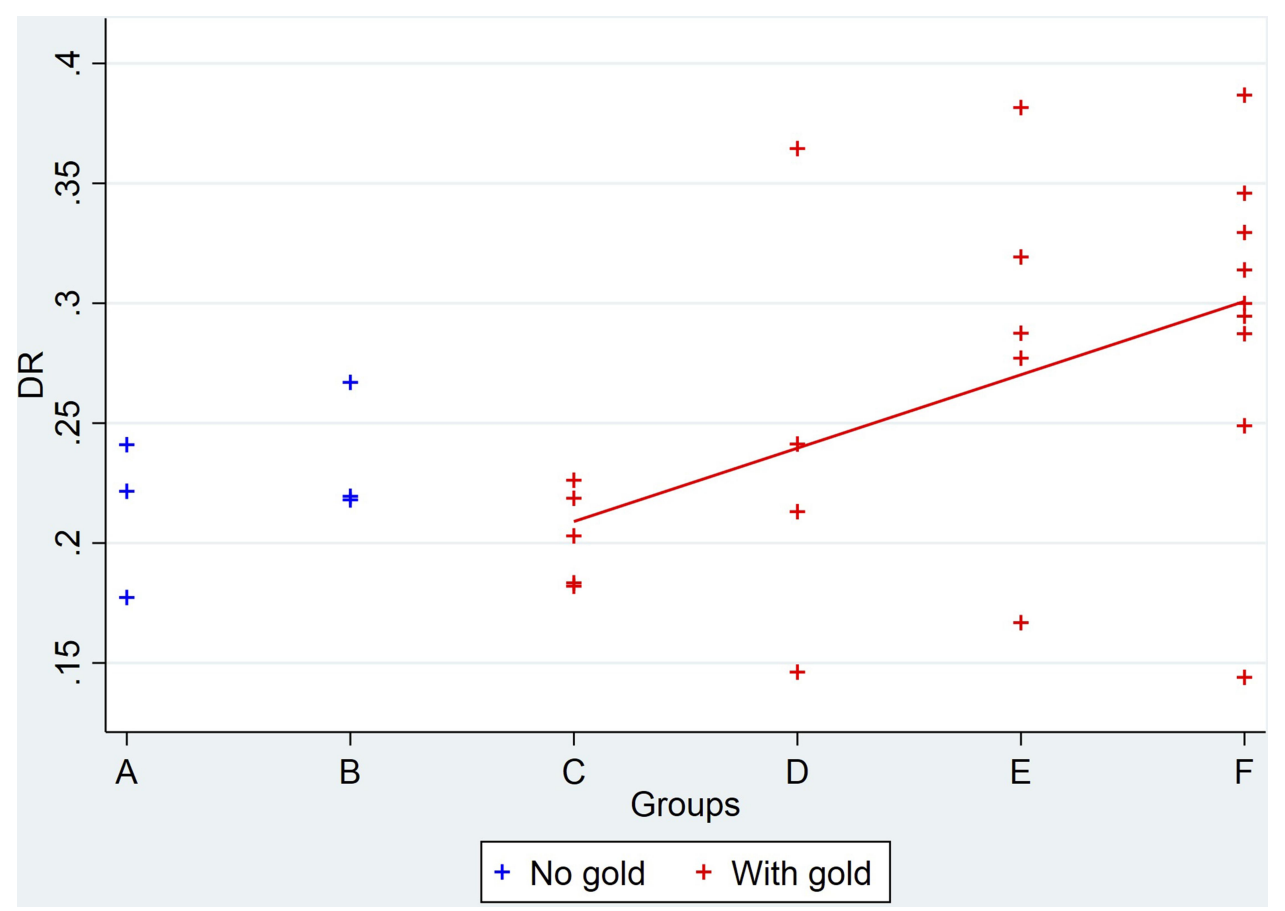

Figure 4 DR measurements of the study group. The highest absolute DR slope values were recorded in rats with cancer (group F) and in rats with moderate to severe dysplasia (group E). The lowest values were recorded in rats with hyperkeratosis and mild dysplasia and in all of the control rats reflecting the low concentration of EGFR. The oblique line represents the trend; a significant trend of the DR measurements in the posterior area correlated with the progression of the severity of the lesions (Cuzick trend test, $\mathrm{P}=0.018)$.

\section{Statistical Analysis}

Comparison of the DR values in the posterior tongue between the three control groups $\mathrm{A}, \mathrm{B}$, and $\mathrm{C}$ did not reveal any statistical differences (Kruskal-Wallis $\mathrm{p}=0.4862$ ). The DR values in healthy rats with injected C-GNRs (group C) did not differ from healthy rats without injected C-GNRs (group A) (exact MW p=0.79) and the absorption of light was even slightly less in group $\mathrm{A}$ than that in group $\mathrm{C}$ (Exact MW p=0.25).

DR measurements following intravenous injection of C-GNRs revealed a significant increase in light absorption in rats with cancer (group F) compared with rats in group $\mathrm{C}(\mathrm{p}<0.02$, sensitivity $100 \%$, specificity $89 \%$ ) (Figure 4 ).

A significant trend of the DR measurements in the posterior area correlated with the progression of the severity of the lesions (Cuzick trend test, $\mathrm{P}=0.018$ ) (Figure 4). ROC analysis revealed $\mathrm{AUC}=0.79(95 \% \mathrm{CI}=(0.56,1.0))$. A significant difference was found between high-risk rats (groups E, F) and low-risk rats (groups C, D); The best cut-point for discrimination was defined as 0.245 and the corresponding sensitivity was $86 \%$ and specificity $89 \%$. DOR=48 (Table 3 ).

The differences in the DR measurements between the posterior and anterior parts were found only in the cancerous group $\mathrm{F}$ (Wilcoxon $\mathrm{p}=0.0039$ ). In all other groups, no differences were encountered due to the presence of dysplasia throughout the entire tongue epithelium.

\section{Discussion}

Most oral cancer cases are discovered in advance stage; therefore, secondary prevention is the key to prevention, which means detecting cancers in early enough stage that they can be treated. Several adjunctive tests have been applied for augmenting the diagnosis, such as cytological

Table 3 Statistical Analyses for Comparison Between the High and Low Risk Groups (C, D versus E, F)

\begin{tabular}{|l|c|c|c|}
\hline \multirow{2}{*}{ Risk/Group } & \multicolumn{2}{|c|}{$\begin{array}{c}\text { Number of Rats/Diffusion } \\
\text { Reflection }\end{array}$} & Total \\
\cline { 2 - 3 } & DR<0.245 & DR $\geq \mathbf{0 . 2 4 5}$ & \\
\hline Low risk (C, D) & 8 & 1 & 9 \\
& 88.98 & $1 \mathrm{I} .1 \mathrm{I}$ & 100.00 \\
\hline High risk (E, F) & 2 & 12 & 14 \\
& 14.29 & 85.71 & 100.00 \\
\hline Total & 10 & 13 & 23 \\
& 43.48 & 56.52 & 100.00 \\
\hline
\end{tabular}


testing, autofluorescence, and tissue reflectance methods, nevertheless, the potential benefit of these devices for cancer diagnosis in the oral cavity is debated. ${ }^{11}$ Realtime optical diagnostic methods, such as Raman spectroscopy, elastic scattering spectroscopy, diffuse reflectance spectroscopy, narrow-band imaging, and confocal reflectance microscopy emerged to distinguish malignant from benign oral lesions, ${ }^{10}$ however, these methods are still under investigation and are yet of limited clinical use.

The present study shows the potential of the DR optical method to detect OSCC specifically targeted by systemically delivered C-GNR. OSCC was identified in all rats with cancer compared to control rats with $89 \%$ sensitivity and $100 \%$ specificity. In addition, a significant difference was found between rats with high-risk lesions (groups E, F) compared with low-risk rats (groups C, D) (sensitivity $86 \%$ specificity $89 \%$ ). Discriminating low-risk lesions (hyperkeratosis and mild dysplasia) from high-risk lesions (moderate to severe dysplasia and OSCC) is of utmost importance as these lesions are prone to transform into invasive cancer. ${ }^{10,35}$ DR measurements taken from rats with dysplastic lesions show a significant trend; increase in the absorption of light with the increase of the dysplastic severity and dramatic increase once cancer is detected (Cuzick trend test, Prob $=0.018$ ). These results are in accordance with our previous in vitro study using hyperspectral microscopy on paraffin-embedded tissues, showing an increase in the reflectance intensity with the progression of the disease, lowest in the control group and increasing as the dysplastic changes increase. ${ }^{17}$ Additional support came from the study of Ankri et al using airSEM microscopy on OSCC slides, ${ }^{22}$ showing a gradient decrease of C-GNRs concentration from the tumor to normal epithelium. Specifically targeting high concentration of C-GNRs exclusively to oral tumor cells and the DR-nanophotonic detection of these nanoparticles from the surface of the tongue makes the presented method unique for early detection of OSCC. An interesting observation is the differences between the anterior and posterior tongue, the main statistical differences were found in the carcinoma cases and not in the dysplastic cases. These results support the field cancerization theory as the entire tongue is affected by the carcinogen.

For efficient targeting and imaging, several aspects are to be considered. The nanoparticles should have the best physical design to enhance imaging, the biology of the tumor to be targeted, and the coating moieties that will confer the best specific active binding potential. ${ }^{2,6}$ In addition, biocompatibility and cytotoxicity are factors to be considered.

GNRs sized $15 \times 50 \mathrm{~nm}$, were found to be highly feasible for the study because of the maximum absorption and scattering properties at the red to infrared light spectrum. ${ }^{17,22,24}$ Size is known to affect the distribution of the nanoparticles, the smallest GNPs revealed the most widespread distribution. ${ }^{36-38}$

Tumor penetration through the vasculature is the first barrier for nanoparticles to gain access to the tumor microenvironment. The enhanced permeability and retention (EPR) effect is responsible for passive GNPs accumulation in the microenvironment. ${ }^{5}$ Recent study found that passive extravasation contributed only a fraction of the nanoparticle tumor accumulation, and up to $97 \%$ of nanoparticles use an active process through trans-endothelial pathways. $^{39}$

Active targeting, the result of specific binding to cancer cells, enhances the retention of the nanoparticles in the tumor environment. ${ }^{2,27}$ The use of specific ligands as binding molecules relies on the fact that several receptors are frequently overexpressed in solid tumors. ${ }^{40}$ Head and neck cancers including oral cancer exhibit an increased EGFR expression. ${ }^{18-21}$ Specific binding of gold nanorods to oral cancer cells is achieved by conjugating with Cetuximab, a chimeric monoclonal mouse-human immunoglobulin G1 (IgG1) antibody. Cetuximab binds specifically to the extracellular ligand-binding domain of EGFR with a binding affinity that is two log units greater than that of EGF or TGF- $\alpha .^{41,42}$ The hyperspectral results demonstrate the presence of increased concentration of C-GNRs in the area related to the tumor (Figure 3).

GNPs have an excellent stability and biocompatibility, tailorable shapes and sizes, an easily functionalized surface, high drug-loading capacity, and low toxicity. ${ }^{43}$ These properties considered GNPs as a potential tool for diagnosis, imaging and treatment of various cancers, including oral cancer. ${ }^{5-7,27,35,44,45}$ Nevertheless, despite all these benefits, biocompatibility and cytotoxicity of the GNPs is still a factor to be considered. Several assays have been used to study the toxicity of GNPs in biological systems presenting a clear size-dependence. ${ }^{46}$ To reduce toxicity, GNPs are coated with PEG rendering the nanoparticles perfectly soluble in water and minimizes any protein adsorption. ${ }^{43}$

Even though basic research studies have revealed a greater promise of nanomaterial to improve prognosis, none was translated into clinical benefits for oral cancer patients. $^{7}$ Accurate and early detection of OSCC and high- 
grade dysplastic lesions is a critical challenge. The superficial presence of OSCC and the overexpression of EGFR make these tumors ideal for the use of nanophotonic-based detection. The use of gold nanoparticles combined with nanophononics for in vivo diagnosis of OSCC suggests a promising potential for oral cancer detection. In addition, providing a significant support for the use of C-GNRs for selective drug delivery.

\section{Acknowledgments}

The authors thank Hana Vered for her technical support in preparing the histologic slides.

\section{Author Contributions}

All authors made a significant contribution to the work reported, whether that is in the conception, study design, execution, acquisition of data, analysis and interpretation, or in all these areas; took part in drafting, revising or critically reviewing the article; gave final approval of the version to be published; have agreed on the journal to which the article has been submitted; and agree to be accountable for all aspects of the work. Contributed equally as senior authors: Dror Fixler and Abraham Hirshberg.

\section{Funding}

This work was supported by the Israel Science Foundation [grant numbers 1760/16]. Role of the funding source: The funding source had no involvement in study design; in the collection, analysis and interpretation of data; in the writing of the report; and in the decision to submit the article for publication.

\section{Disclosure}

The authors report no conflicts of interest for this work.

\section{References}

1. Song S, Hao Y, Yang X, Patra P, Chen J. Using gold nanoparticles as delivery vehicles for targeted delivery of chemotherapy drug fludarabine phosphate to treat hematological cancers. $J$ Nanosci Nanotechnol. 2016;16(3):2582-2586. doi:10.1166/jnn.2016.12349

2. Biffi S, Voltan R, Bortot B, Zauli G, Secchiero P. Actively targeted nanocarriers for drug delivery to cancer cells. Expert Opin Drug Deliv. 2019;16:481-496. doi:10.1080/17425247.2019.1604679

3. Rosenblum D, Joshi N, Tao W, Karp JM, Peer D. Progress and challenges towards targeted delivery of cancer therapeutics. Nat Commun. 2018;12:1410. doi:10.1038/s41467-018-03705-y

4. Aminabad NS, Farshbaf M, Akbarzadeh A. Recent advances of gold nanoparticles in biomedical applications: state of the art. Cell Biochem Biophys. 2019;77:123-137. doi:10.1007/s12013-018-0863-4
5. Grodzinski P, Kircher M, Goldberg M, Gabizon A. Integrating nanotechnology into cancer care. ACS Nano. 2019;13:7370-7376. doi:10.1021/acsnano.9b04266

6. Chen XJ, Zhang XQ, Liu Q, Zhang J, Zhou G. Nanotechnology: a promising method for oral cancer detection and diagnosis. J Nanobiotechnology. 2018;16(1):52. doi:10.1186/s12951-018-0378-6

7. de Lima JM, Bonan PR, da Cruz Perez DE, Hier M, Alaoui-Jamali MA, da Silva SD. Nanoparticle-based chemotherapy formulations for head and neck cancer: a systematic review and perspectives. Nanomaterials. 2020;10(10):1938. doi:10.3390/nano10101938

8. Bray F, Ferlay J, Soerjomataram I, Siegel RL, Torre LA, Jemal A. Global cancer statistics 2018: GLOBOCAN estimates of incidence and mortality worldwide for 36 cancers in 185 countries. CA Cancer J Clin. 2018;1-31.

9. Siegel RL, Miller KD, Jemal A. Cancer statistics, 2019. CA Cancer J Clin. 2019;69:7-34. doi:10.3322/caac.21551

10. Lingen MW, Abt E, Agrawal N, et al. Evidence-based clinical practice guideline for the evaluation of potentially malignant disorders in the oral cavity: a report of the American Dental Association. $J$ Am Dent Assoc. 2017;148:712-727. doi:10.1016/j.adaj.2017.07.032

11. Macey R, Walsh T, Brocklehurst P, et al. Diagnostic tests for oral cancer and potentially malignant disorders in patients presenting with clinically evident lesions. Cochrane Database Syst Rev. 2015;5: CD010276.

12. Kim CS, Wilder-Smith P, Ahn YC, Liaw LH, Chen Z, Kwon YJ. Enhanced detection of early-stage oral cancer in vivo by optical coherence tomography using multimodal delivery of gold nanoparticles. J Biomed Opt. 2009;14:034008. doi:10.1117/1.3130323

13. Shanavas A, Sasidharan S, Bahadur D, Srivastava R. Magnetic core-shell hybrid nanoparticles for receptor targeted anti-cancer therapy and magnetic resonance imaging. $J$ Colloid Interface Sci. 2017;486:112-120. doi:10.1016/j.jcis.2016.09.060

14. Luke GP, Myers JN, Emelianov SY, Sokolov KV. Sentinel lymph node biopsy revisited: ultrasound-guided photoacoustic detection of micrometastases using molecularly targeted plasmonic nanosensors. Cancer Res. 2014;74(19):5397-5408. doi:10.1158/0008-5472.CAN14-0796

15. Kang JW, So PTC, Dasari RR, Lim DK. High resolution live cell raman imaging using subcellular organelle-targeting SERS-sensitive gold nanoparticles with highly narrow intra-nanogap. Nano Lett. 2015;15:1766-1772. doi:10.1021/n1504444w

16. Ankri R, Fixler D. Gold nanorods based diffusion reflection measurements: current status and perspectives for clinical applications. Nanophotonics. 2017;6:1031-1042. doi:10.1515/nanoph-2017-0033

17. Hirshberg A, Allon I, Novikov I, Ankri R, Ashkenaz A, Fixler D. Gold-nanorods reflectance discriminate benign from malignant oral lesions. Nanomedicine. 2017;13(4):1333-1339. doi:10.1016/j. nano.2017.01.003

18. Johnson DE, Burtness B, Leemans CR, Lui VWY, Bauman JE, Grandis JR. Head and neck squamous cell carcinoma. Nat Rev Dis Primers. 2020;6(1):92.

19. Zhu X, Zhang F, Zhang W, He J, Zhao Y, Chen X. Prognostic role of epidermal growth factor receptor in head and neck cancer: a meta-analysis. J Surg Oncol. 2013;108(6):387-397. doi:10.1002/jso.23406

20. Rubin Grandis J, Melhem MF, Gooding WE, et al. Levels of TGF-alpha and EGFR protein in head and neck squamous cell carcinoma and patient survival. J Natl Cancer Inst. 1998;90(11):824-832. doi:10.1093/jnci/90.11.824

21. Rajaram P, Chandra P, Ticku S, Pallavi BK, Rudresh KB, Mansabdar P. Epidermal growth factor receptor: role in human cancer. Indian J Dent Res. 2017;28(6):687-694. doi:10.4103/ijdr. IJDR_534_16

22. Ankri R, Ashkenazy A, Milstein Y, et al. Gold nano rods based airSEM and diffusion reflection imaging for mapping tumor margins in SCC cells. ACS Nano. 2016;10(2):2349-2356. doi:10.1021/ acsnano. $5 \mathrm{~b} 07114$ 
23. Dayan D, Hirshberg A, Kaplan I, Rotem N, Bodner L. Experimental tongue cancer in desalivated rats. Oral Oncol. 1997;33(2):105-109. doi:10.1016/S0964-1955(96)00048-6

24. Fixler D, Ankri R, Kaplan I, Novikove I, Hirshberg A. Diffusion reflection: a novel method for detection of oral cancer. J Dent Res. 2014;93(6):602-606. doi:10.1177/0022034514529973

25. Ohne M, Satoh T, Yamada S, Takai H. Experimental tongue carcinoma of rats induced by oral administration of 4- nitroquinoline 1-oxide (4NQO) in drinking water. Oral Surg Oral Med Oral Pathol. 1985;59(6):600-607. doi:10.1016/0030-4220(85)90189-6

26. Menon JU, Jadeja P, Tambe P, Vu K, Yuan B, Nguyen KT. Nanomaterials for photo-based diagnostic and therapeutic applications. Theranostics. 2013;3(3):152-166. doi:10.7150/ thno. 5327

27. Singh P, Pandit S, Mokkapati VRSS, Garg A, Ravikumar V, Mijakovic I. Gold nanoparticles in diagnostics and therapeutics for human cancer. Int J Mol Sci. 2018;19(7):1979. doi:10.3390/ ijms 19071979

28. Dai Q, Wilhelm S, Ding D, et al. Quantifying the ligand-coated nanoparticle delivery to cancer cells in solid tumors. ACS Nano. 2018;12(8):8423-8435. doi:10.1021/acsnano.8b03900

29. Toy R, Peiris PM, Ghaghada KB, Karathanasis E. Shaping cancer nanomedicine: the effect of particle shape on the in vivo journey of nanoparticles. Nanomedicine. 2014;9(1):121-134.

30. Ankri R, Taitelbaum H, Fixler D. Reflected light intensity profile of two-layer tissues: phantom experiments. J Biomed Opt. 2011;16 (8):085001. doi:10.1117/1.3605694

31. Ankri R, Duadi H, Motiei M. In-vivo tumor detection using diffusion reflection measurements of targeted gold nanorods - a quantitative study. J Biophotonics. 2012;5(3):263-273. doi:10.1002/ jbio. 201100120

32. Fixler D, Ankri R. Subcutaneous gold nanorods detection with diffusion reflection measurement. J Biomed Opt. 2013;18(6):61226. doi:10.1117/1.JBO.18.6.061226

33. Ankri R, Chakraborty R, Motiei M, Fixler D. Three-dimensional highly sensitive diffusion reflection-based imaging method for the in vivo localization of atherosclerosis plaques following gold nanorods accumulation. ACS Omega. 2018;3(6):6134-6142. doi:10.1021/ acsomega.8b00750

34. Ankri R, Taitelbaum H, Fixler D. On phantom experiment of the photon migration model in tissues. Open Opt J. 2011;5:28-32. doi:10.2174/1874328501105010028
35. Ranganathan K, Kavitha L. Oral epithelial dysplasia: classifications and clinical relevance in risk assessment of oral potentially malignant disorders. J Oral Maxillofac Pathol. 2019;23(1):19-27. doi:10.4103/ jomfp.JOMFP_13_19

36. Zein R, Sharrouf W, Selting K. Physical properties of nanoparticles that result in improved cancer targeting. $J$ Oncol. 2020;2020:5194780. doi:10.1155/2020/5194780

37. Kumar D, Saini N, Jain N, Sareen R, Pandit V. Gold nanoparticles: an era in bionanotechnology. Expert Opin Drug Deliv. 2013;10 (3):397-409. doi:10.1517/17425247.2013.749854

38. Lopez-Chaves C, Soto-Alvaredo J, Montes-Bayon M, Bettmer J, Llopis J, Sanchez-Gonzalez C. Gold nanoparticles: distribution, bioaccumulation and toxicity. In vitro and in vivo studies. Nanomedicine. 2018;14(1):1-12. doi:10.1016/j.nano.2017.08.011

39. Sindhwani S, Syed AM, Ngai J, et al. The entry of nanoparticles into solid tumours. Nat Mater. 2020;19(5):566-575. doi:10.1038/s41563019-0566-2

40. Glasgow MDK, Chougule MB. Recent developments in active tumor targeted multifunctional nanoparticles for combination chemotherapy in cancer treatment and imaging. J Biomed Nanotechnol. 2015;11 (11):1859-1898. doi:10.1166/jbn.2015.2145

41. Benchekroun MT, Saintigny P, Thomas SM, El-Naggar AK, Papadimitrakopoulou V. Epidermal growth factor receptor expression and gene copy number in the risk of oral cancer. Cancer Prev Res. 2010;3(7):800-809. doi:10.1158/1940-6207.CAPR-09-0163

42. Mahtani RL, Macdonald JS. Synergy between cetuximab and chemotherapy in tumors of the gastrointestinal tract. Oncologist. 2008;13 (1):39-50. doi:10.1634/theoncologist.2006-0049

43. Connor DM, Broome A-M. Gold nanoparticles for the delivery of cancer therapeutics. Adv Cancer Res. 2018;139:163-184.

44. Movia D, Gerard V, Maguire CM, et al. A safe-by-design approach to the development of gold nanoboxes as carriers for internalization into cancer cells. Biomaterials. 2014;35(9):2543-2557. doi:10.1016/j. biomaterials.2013.12.057

45. Essawy MM, El-Sheikh SM, Raslan HS, et al. Function of gold nanoparticles in oral cancer beyond drug delivery: implications in cell apoptosis. Oral Dis. 2020. doi:10.1111/odi.13551

46. Deng J, Yao M, Gao C. Cytotoxicity of gold nanoparticles with different structures and surface-anchored chiral polymers. Acta Biomater. 2017;53:610-618. doi:10.1016/j.actbio.2017.01.082
International Journal of Nanomedicine

\section{Publish your work in this journal}

The International Journal of Nanomedicine is an international, peerreviewed journal focusing on the application of nanotechnology in diagnostics, therapeutics, and drug delivery systems throughout the biomedical field. This journal is indexed on PubMed Central, MedLine, CAS, SciSearch ${ }^{\circledR}$, Current Contents ${ }^{\mathbb{R}} /$ Clinical Medicine,
Journal Citation Reports/Science Edition, EMBase, Scopus and the Elsevier Bibliographic databases. The manuscript management system is completely online and includes a very quick and fair peer-review system, which is all easy to use. Visit http://www.dovepress.com/ testimonials.php to read real quotes from published authors. 